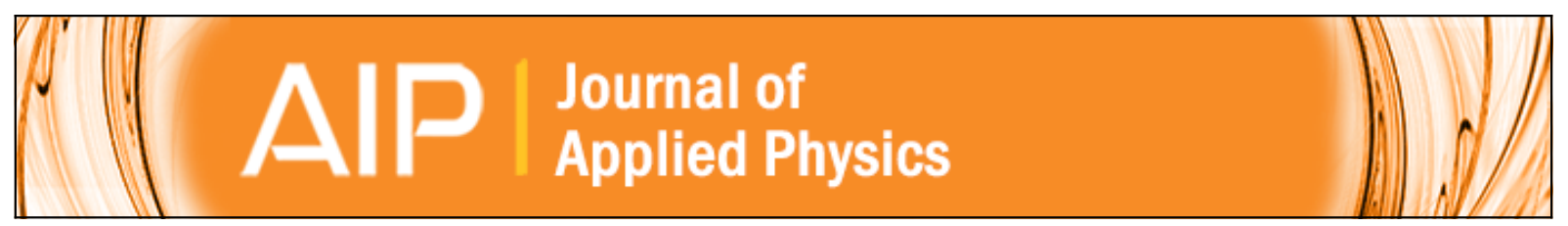

\title{
Electrically detected magnetic resonance modeling and fitting: An equivalent circuit approach
}

D. M. G. Leite, A. Batagin-Neto, O. Nunes-Neto, J. A. Gómez, and C. F. O. Graeff

Citation: Journal of Applied Physics 115, 034510 (2014); doi: 10.1063/1.4862178

View online: http://dx.doi.org/10.1063/1.4862178

View Table of Contents: http://scitation.aip.org/content/aip/journal/jap/115/3?ver=pdfcov

Published by the AIP Publishing

\section{Articles you may be interested in}

Equivalent circuit models for the analysis of electric response and magnetic response of compact triangular electromagnetic resonator

J. Appl. Phys. 113, 044905 (2013); 10.1063/1.4789797

A generalized equivalent circuit theory for the electric and magnetic resonances of metallic wire networks

J. Appl. Phys. 105, 113121 (2009); 10.1063/1.3143037

A compact equivalent circuit for the dark current-voltage characteristics of nonideal solar cells

J. Appl. Phys. 100, 084513 (2006); 10.1063/1.2357641

Equivalent circuit model for carbon nanotube Schottky barrier: Influence of neutral polarized gas molecules Appl. Phys. Lett. 88, 083106 (2006); 10.1063/1.2177356

Deposition and $1.54 \mu \mathrm{m} \mathrm{Er} \mathrm{3+} \mathrm{luminescent} \mathrm{properties} \mathrm{of} \mathrm{erbium-doped} \mathrm{hydrogenated} \mathrm{amorphous} \mathrm{silicon} \mathrm{thin}$ films by electron cyclotron resonance plasma enhanced chemical vapor deposition of $\mathrm{SiH} 4$ with concurrent sputtering of erbium

J. Vac. Sci. Technol. A 17, 3230 (1999); 10.1116/1.582047

\section{AIP $\mid$ chaos CALL FOR APPLICANTS Seeking new Editor-in-Chief}




\title{
Electrically detected magnetic resonance modeling and fitting: An equivalent circuit approach
}

\author{
D. M. G. Leite, ${ }^{1, a)}$ A. Batagin-Neto, ${ }^{2}$ O. Nunes-Neto, ${ }^{2}$ J. A. Gómez, ${ }^{3}$ and C. F. O. Graeff ${ }^{2,4}$ \\ ${ }^{1}$ UNIFEI-Universidade Federal de Itajubá, Av. BPS, 1303, 37500-903 Itajubá, MG, Brazil \\ ${ }^{2}$ UNESP_Univ Estadual Paulista, POSMAT_Programa de Pós-Graduação em Ciência e Tecnologia de \\ Materiais, Av. Eng. Luiz Edmundo Carrijo Coube, 14-01, 17033-360 Bauru, SP, Brazil \\ ${ }^{3}$ Departamento de Física, FFCLRP-USP, Av. Bandeirantes 3900, 14040-901 Ribeirão Preto, SP, Brazil \\ ${ }^{4}$ DF-FC, UNESP_Univ Estadual Paulista, Av. Eng. Luiz Edmundo Carrijo Coube, 14-01, 17033-360 Bauru, \\ SP, Brazil
}

(Received 10 December 2013; accepted 1 January 2014; published online 17 January 2014)

\begin{abstract}
The physics of electrically detected magnetic resonance (EDMR) quadrature spectra is investigated. An equivalent circuit model is proposed in order to retrieve crucial information in a variety of different situations. This model allows the discrimination and determination of spectroscopic parameters associated to distinct resonant spin lines responsible for the total signal. The model considers not just the electrical response of the sample but also features of the measuring circuit and their influence on the resulting spectral lines. As a consequence, from our model, it is possible to separate different regimes, which depend basically on the modulation frequency and the RC constant of the circuit. In what is called the high frequency regime, it is shown that the sign of the signal can be determined. Recent EDMR spectra from $\mathrm{Alq}_{3}$ based organic light emitting diodes, as well as from a-Si:H reported in the literature, were successfully fitted by the model. Accurate values of $g$-factor and linewidth of the resonant lines were obtained. (C) 2014 AIP Publishing LLC. [http://dx.doi.org/10.1063/1.4862178]
\end{abstract}

\section{INTRODUCTION}

Electronic magnetic resonance (EMR) is a powerful tool widely employed in material research and device characterization. In general, these techniques are based on detecting changes in a given observable when electron spin resonance (ESR) condition is reached and allows the investigation of intrinsic properties of charge carrier's spin, as well as its interaction with the local environment. ${ }^{1-4}$ ESR, electrically detected magnetic resonance (EDMR), and optically detected magnetic resonance (ODMR) are the most representatives EMR techniques. ${ }^{5-11}$

In what concerns electronic device characterization EDMR is the most suitable since experiments can be done in real devices under working conditions. ${ }^{12-14}$ It consists of detecting electrical conductivity changes induced by spin resonance. During charge transport or recombination, paramagnetic or ESR active spin pairs are commonly formed in the device. Since transitions involving these centers, called often precursor pairs, are governed by spin selection rules, measurable changes in conductivity can be induced by electron magnetic resonance, resulting in an EDMR signal. In this sense, it is a very sensitive and selective tool to investigate transport and recombination in different material and electronic devices.

Indeed, different spin-dependent processes (SDPs) can be measured by EDMR. They have been commonly associated to conduction electron scattering, tunneling, trapping, and recombination. ${ }^{15}$ In the particular case of disordered and organic materials, these mechanisms have been discussed in terms of

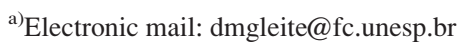

bipolarons and excitons formation, ${ }^{13,16,17}$ polaronic and excitonic recombination, ${ }^{15,18}$ or triplet-triplet annihilation. ${ }^{19}$

Since these processes involve distinct precursor pairs formed from electrons, holes, or excitons, which experience different nanoscopic environments, it is expected that they present dissimilar spectroscopic characteristics. In this sense, EDMR spectra can be considered as a complex combination of distinct resonant spin lines (RSL).

Dersch et al. ${ }^{20}$ were one of the first to explore the use of phase analysis of continuous wave EDMR (for simplicity EDMR from here on) signal, in order to discriminate RSLs in a-Si:H. By tuning the detection phase, they identified and isolated two RSLs attributed to electron tunneling from tail states to dangling bonds and holes thermalization to doubly occupied dangling bonds. After that, many other research groups have explored phase analysis in order to extract more and potentially better physical information from EDMR experiments. $^{21-25}$

Recently, Lee et al. ${ }^{26}$ presented a thorough study on modulation frequency dependence of continuous-wave optically/electrically detected magnetic resonance experiments. It was demonstrated that the phase response of the device is strongly dependent on intrinsic transition rates of the RSL, as well as on the experimental parameters employed during data acquisition. In their work, the relative intensity of inphase and out-of-phase components of the EDMR signal were evaluated using rate equations. Nevertheless, their approach did not permit lineshape analysis and its use on the discrimination of different RSL as will be discussed here. Wimbauer et al..$^{27}$ proposed a RC equivalent circuit in order to simulate the amplitude and phase of the various components of the EDMR spectra associated to inorganic 
multilayer devices. Again in this approach, the lineshape and $\mathrm{g}$-factor were not addressed.

In the present study, we extend Wimbauer's model, for this purpose, circuit elements are used to model lineshapes and $g$-factors from the different RSLs. The magnetic field modulation effects are an integral part of the description, which allows the discrimination of individual RSL signals by phase analysis. The work is organized as follow. In Sec. II, the equivalent circuit model employed and phase detection details are presented, followed by Sec. III where a brief discussion of some implications of the circuit model, as well as examples regarding out-of-phase signals are presented. In Sec. IV, the methodology is applied to experimental results found in the literature. Finally, in Sec. V, the main conclusions are presented. In addition, line-broadening effects induced by excessive magnetic field modulation are discussed in Appendix A; and in Appendix B, fitting software based on the proposed circuit model is presented.

\section{THE CIRCUIT MODEL}

EDMR spectra are ordinarily obtained by monitoring conductivity changes by phase sensitive detection (PSD). For that purpose, the magnetic field is modulated, creating a small AC in the measuring circuit at the resonance condition, which is much smaller than the sample DC, typically $\mathrm{AC} \leq 10^{-4} \mathrm{DC}$.

Given the sample's characteristics and the measuring circuit used, the EDMR setup configures an electrical circuit with a variety of elements that in most cases can be, in a first approximation described as a RC circuit, thus a low-pass filter. When double channel Lock-in amplifier is used the output signal is divided in two channels, $X$ and $Y$ in phase quadrature. It is common practice that the signal in the $X$ channel is maximized and used as the $0^{\circ}$ detection phase. Consequently, the signal in the $Y$ channel (in $90^{\circ}$ ) is zero or very small. The main contribution of this work is to provide a clear interpretation of $Y$ channel signals.

\section{A. The equivalent circuit}

The use of equivalent circuits is an efficient way of obtaining relevant physical information from complex systems. For example, it can be used to simulate the impedance response of double or multi junction devices, such as light emitting diodes (LEDs), organic LEDs (OLEDs), and solar cells. $^{28-31}$

In our model, the sample or device is described as a parallel RC circuit with a constant capacitance $C_{S}$ and a rheostat $R$ that depends on the applied magnetic field $H$. The dependence of $R(H)$ basically emulates the conductivity changes under resonance as will become clearer in the following discussion. Figure 1(a) shows the schematic representations of the proposed equivalent circuit. $V_{0}$ is the external applied voltage; $R_{M}$ is the measuring resistance used to convert the current into measured voltage $V_{M}$. $C_{C}$ is the circuit capacitance, mainly due to cables and connections.

Figure 1(b) shows the simplified version of the proposed equivalent circuit by converting the sample resistance $R$ to conductance $G=1 / R$, and associating the sample capacitance

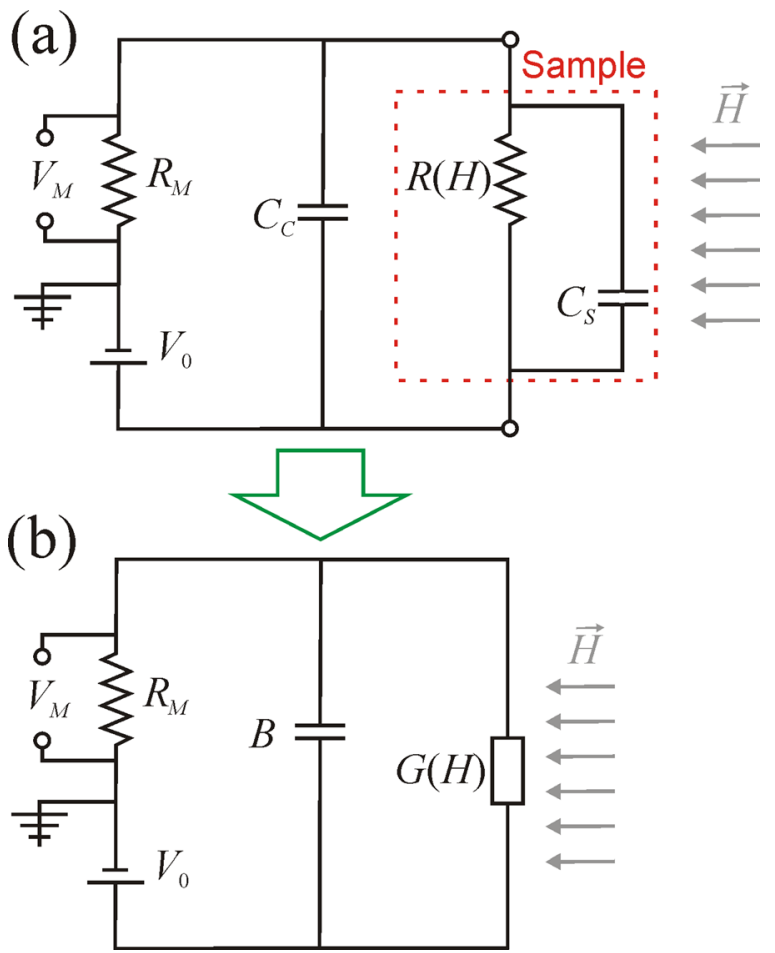

FIG. 1. (a) Squematic representation of the real measuring circuit with sample as equivalent parallel RC circuit and (b) the correspondent simplified version used to develop the model.

$\left(C_{S}\right)$ with the measuring circuit capacitance $\left(C_{C}\right)$ as the equivalent capacitance $C=C_{S}+C_{C}$, which is converted to the circuit susceptance $B=\omega C$. Here, $\omega$ stands for the angular frequency of the modulation induced AC. Notice that the AC is observed only close to resonance.

In the proposed model, $G$ has a constant term $G_{0}$ plus a magnetic field dependent term represented, for simplicity, by a pseudo-Voigt profile function

$$
\begin{gathered}
\Omega=\frac{H-H_{R E S}}{\Delta H_{1 / 2}}, \text { and } \\
H_{R E S}=\frac{h v}{\mu_{B} g},
\end{gathered}
$$$$
G=G_{0}+G_{H}\left[\alpha\left(2^{-4 \Omega^{2}}\right)+(1-\alpha)\left(\frac{1}{1+4 \Omega^{2}}\right)\right], \text { with }
$$

where $\Delta H_{1 / 2}$ represents the full width at half maximum of the resonant line, $h v$ is the microwave energy, $\mu_{B}$ is the Bohr magneton, $g$ is the $g$-factor, and $G_{H}$ is the maximum conductance shift (positive or negative) in the resonant condition. Equation (1a) has a Gaussian and Lorentzian term weighted by $\alpha$. Notice that our Gaussian term has an unusual representation since we have changed the basis from neperian to 2 , just to simplify the expression.

In an EDMR experiment, usually $h \nu$ is constant so the magnetic field is swept linearly around a central field $H_{0}$ close to the resonant condition. As discussed previously, a modulation field with angular frequency $\omega=2 \pi f$ and 
amplitude $H_{m}$ is used for PSD resulting in a modulated magnetic field given by

$$
\begin{gathered}
H(t)=\underbrace{H_{0}+\Delta H\left(\frac{t}{\Delta t}-\frac{1}{2}\right)}_{H_{L}}+\underbrace{H_{m} \sin (\omega t)}_{H_{M O D}}, \\
H=H_{L}+H_{M O D},
\end{gathered}
$$

where $\Delta H$ and $\Delta t$ represent the amplitude of the field sweep and the sweep time, respectively. For simplicity, we define $H_{L}$ as the field component, which is swept, and $H_{M O D}$ as the modulated component. The time dependence of $H_{L}$ and $H_{M O D}$ was omitted in the second equation for simplicity.

\section{B. Signal processing and detection}

It is easy to realize that $G$ has no simple time dependence, and thus it is convenient to use Fourier expansion

$$
G(t) \approx c_{0}+c_{1} \sin (\omega t+\phi)+\Lambda,
$$

where $c_{i}$ are the Fourier coefficients and $\Lambda$ stands for the higher frequency terms. Here, we define $\phi$ as a constant phase shift from the reference signal.

As in the exhaustively studied ESR case, the Fourier coefficients can be determined via Taylor expansion. ${ }^{32}$ For low values of $H_{m}$ in comparison to $\Delta H_{1 / 2}\left(H_{m} / \Delta H_{1 / 2} \ll 1\right)$, which is the most common situation in EDMR and ESR experiments, it is possible to use the following approximations for the dependence of $c_{0}$ and $c_{1}$ with $H_{L}$ :

$$
\begin{gathered}
c_{0}\left(H_{L}\right) \approx G\left(H_{L}\right), \\
\left.c_{1}\left(H_{L}\right) \approx \frac{H_{m}}{2} \frac{d G}{d H}\right|_{H=H_{L}}=\frac{H_{m}}{2} \frac{d G}{d H}\left(H_{L}\right) .
\end{gathered}
$$

Remember that $c_{1}$ is measured in PSD. Notice that when $H_{m} \sim \Delta H_{1 / 2}$, the approximation of $c_{1}$ given by Eq. (5) is no longer valid, see Appendix A.

For $R_{M} \ll R$, which is normally the case, the DC component of $V_{M}$ can be approximated to

$$
V_{D C}\left(H_{L}\right)=V_{0} R_{M} G\left(H_{L}\right) .
$$

While the output signal of the PSD can be written as

$$
V_{A C}\left(H_{L}\right)=S\left(H_{L}\right) \angle \Phi\left(H_{L}\right)
$$

where

$$
\Phi\left(H_{L}\right)=\phi+\tan ^{-1}\left(B / G\left(H_{L}\right)\right)
$$

represents the phase of the measured signal, and

$$
S\left(H_{L}\right)=\beta V_{0} R_{M} c_{1}\left(H_{L}\right)
$$

is its magnitude. $S$ can assume either positive or negative value at a given $H_{L}$ due to the definition of $c_{l}$, with $\beta$ representing the attenuation factor of the equivalent RC circuit, given, in a first approximation, by

$$
\beta=\left[1+\left(B / G_{0}\right)^{2}\right]^{-1} .
$$

The quadrature signal decomposed in $X$ and $Y$ channels, for the fundamental frequency, is then given by

$$
\begin{gathered}
X\left(H_{L}\right)=S\left(H_{L}\right) \cos \left(\Phi\left(H_{L}\right)-\theta\right), \\
Y\left(H_{L}\right)=S\left(H_{L}\right) \sin \left(\Phi\left(H_{L}\right)-\theta\right),
\end{gathered}
$$

where $\theta$ is an offset angle.

For the following discussions and applications, we always set the detection angle $\theta$ equal or very close to the average value of $\Phi\left(H_{L}\right)$ over the whole $H_{L}$ sweep. In this way, the EDMR signal is mainly in the $X$ channel, with a small component at the $Y$ channel.

\section{DISCUSSION}

The microscopic origin of the EDMR signal with nonvanishing component at $Y$ channel has been discussed in terms of different mechanisms involving, at least, two SDPs or one SDP with a spin pair with different phases. Indeed, SDPs or spin pairs generally experience distinct chemical environment with different spin-orbit or hyperfine couplings as well as spin-lattice relaxation times. These characteristics can lead to EDMR spectra composed by RSL with different resonant fields $\left(H_{R E S}\right)$, different linewidths $\left(\Delta H_{1 / 2}\right)$, and different response phases $(\phi)$.

For example, Dersch et al. ${ }^{20}$ distinguished two independent SDPs in their EDMR study of a-Si:H. A narrow line with $g \sim 2.005$ attributed to spin-dependent recombination of localized band-tail electrons with holes in dangling bonds and a broad line at $g \sim 2.01$ attributed to spin-dependent hopping of localized band-tail holes. These two RSL were found to be dephased by $15^{\circ}$ when using a modulation frequency of $f=1 \mathrm{kHz}$. Notice that for the hopping related signal, the two spins are basically in the same nanoscopic environment in the mobility edge of a-Si:H valence band, while the recombination related signal is coming from distinct spins. The electron is occupying the mobility edge of a$\mathrm{Si}: \mathrm{H}$ conduction band, while the hole is in a dangling bond. Graeff et al. ${ }^{22,25,33}$ separated the contribution of each spin that formed the exciton precursor pair of $\mathrm{Alq}_{3}$ based OLEDs. In this case, only one SDP is responsible for the EDMR signal, and two distinct spins are participating, an electron in the LUMO of $\mathrm{Alq}_{3}$ (or a dopant molecule), and a hole in the $\mathrm{HOMO}$ of $\mathrm{Alq}_{3}$ or $\alpha$-NPD. Contrary to the case of electron recombination in a-Si:H, the two spins give rise to two RSL. The reason why in one case just one RSL is observed and in the other two is not in the scope of this article. However, it is important to notice that this effect may be induced by the microwave energy, or magnetic field used in resonance. As the magnetic field is increased, there is a higher separation in resonant field between spins with different $g$-factors. In this context, Dersch's work was made in X-band, $9 \mathrm{GHz}$, while Graeff's in K-band, $24 \mathrm{GHz}$.

In the following, the influence of $H_{R E S}, \Delta H_{1 / 2}$, and $\phi$ on the simulated quadrature EDMR signal is presented in two 
different regimes depending on the modulation frequency used.

\section{A. Low modulation frequency (LMF)}

In the LMF regime $B / G_{0}<0.01$, which is typically used in EDMR experiments, the signal phase of a single RSL assumes a constant value, $\Phi\left(H_{L}\right) \rightarrow \phi$ in Eq. (8). If only indistinguishable spins are present, for example, in spin dependent hopping, the EDMR signal is solely projected in the $X$ channel, and thus no signal is observed in the $Y$ channel, see Eqs. (11) and (12) when $\theta=\phi$.

However, as discussed previously, the EDMR signal is commonly composed by two or more RSL. Thus, if one considers two independent resonant spin lines, $\mathrm{RSL}^{a}$ and $\mathrm{RSL}^{b}$, responding with different phases, $\phi^{a}$ and $\phi^{b}$ with respect to the reference, a signal will be observed in the $Y$ channel under certain conditions as will be discussed in the following.

In the present circuit model, the presence of two RSLs is represented by splitting the field dependent term in Eq. (1) into two summing terms with independent parameters: $G_{H}{ }^{a}$, $\alpha^{a}, H_{R E S}{ }^{a}$, and $\Delta H_{1 / 2}{ }^{a}$ for the first RSL, and $G_{H}{ }^{b}, \alpha^{b}, H_{R E S}{ }^{b}$, and $\Delta H_{1 / 2}{ }^{b}$ for the second.

Figure 2 shows the simulated quadrature EDMR signals for two representative conditions, in (a) $H_{R E S}{ }^{a}>H_{R E S}{ }^{b}$ with $\Delta H_{1 / 2}{ }^{a}=\Delta H_{1 / 2}{ }^{b}$, and in (b) $\Delta H_{1 / 2}{ }^{a}>\Delta H_{1 / 2}{ }^{b}$ with $H_{R E S}{ }^{a}$ $=H_{R E S}{ }^{b}$. In all cases, $\Delta \phi=1^{\circ}, \alpha^{a}=\alpha^{b}$, and $G_{H}{ }^{a}=G_{H}{ }^{b}>0$. For each condition, seven curves are plotted for $\theta$ varying from $\phi^{a}$ to $\phi^{b}$. Notice that $\theta \approx\left(\phi^{a}+\phi^{b}\right) / 2$ minimizes the signal amplitude in the $Y$ channel, and that the small changes in $\theta$ from $\phi^{a}$ to $\phi^{b}$ does not affect the signal on the $X$ channel.

It is important to emphasize that both cases shown in Fig. 2 are only representative, i.e., it is unlike to have RSLs with exactly the same $g$-factors or same line-widths. However, the quadrature signals of Fig. 2 with $\theta \approx\left(\phi^{a}+\phi^{b}\right) / 2$ are useful to discriminate whether, in real EDMR spectra, the difference on

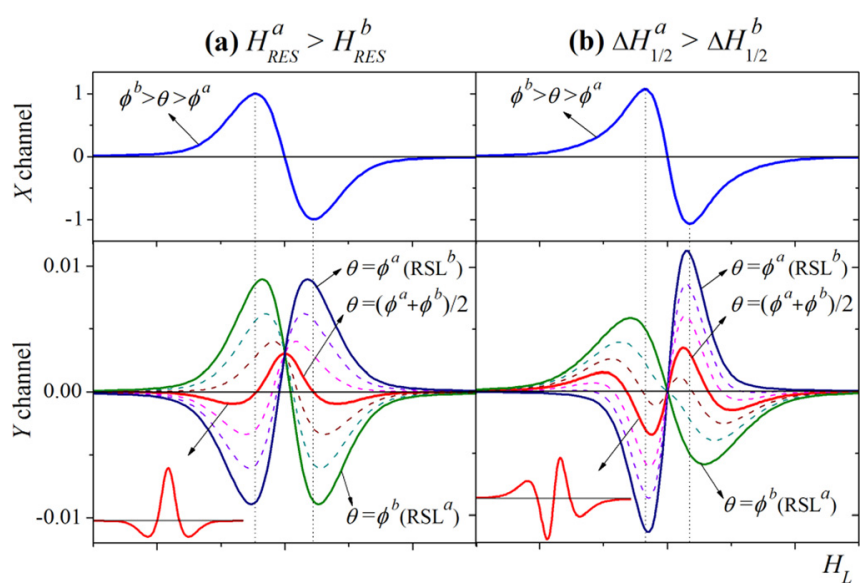

FIG. 2. Simulated EDMR signal in the $X$ and $Y$ channels of a dual phase lock-in amplifier for $G_{H}{ }^{a}=G_{H}{ }^{b}>0, B=0, \alpha^{a}=\alpha^{b}, \Delta \phi=1^{\circ}$, and: (a) $H_{R E S}{ }^{a}>H_{R E S}{ }^{b}$ and $\Delta H_{1 / 2}{ }^{a}=\Delta H_{1 / 2}{ }^{b}$; (b) $H_{R E S}{ }^{a}=H_{R E S}{ }^{b}$ and $\Delta H_{1 / 2}{ }^{a}>\Delta H_{1 / 2}{ }^{b}$. For each condition, seven curves are plotted for $\theta$ varying from $\phi^{a}$ (showing the individual signal of $\mathrm{RSL}^{b}$ in the $Y$ channel) to $\phi^{b}$ (showing the individual signal of $\mathrm{RSL}^{a}$ in the $Y$ channel). Notice that small changes in $\theta$ do not affect the signal in the $X$ channel.
$H_{R E S}$ or on lineshape (represented here by $\Delta H_{1 / 2}$ ) is the dominant effect.

In both cases, described in Fig. 2, the individual RSLs are isolated in the $Y$ channel by shifting $\theta$ from $\phi^{a}$ to $\phi^{b}$ : $\mathrm{RSL}^{b}$ (blue solid line) and $\mathrm{RSL}^{a}$ (green solid line). This procedure was used for example in Refs. 20 and 22. Note also that there is no visible change in the signal in the $X$ channel in all conditions displayed due to the low value of $\Delta \phi=1^{\circ}$. For higher values of $\Delta \phi$ not shown here, significant changes in the $X$ channel are observed.

The special case of Fig. 2(a) with $\theta=\left(\phi^{a}+\phi^{b}\right) / 2$ is reproduced in Fig. 3 in order to elucidate the dependence of the signals in $X$ and $Y$ channels on $\Delta \phi$ (Fig. 3(a)) and $\Delta H_{R E S}=H_{R E S}{ }^{a}-H_{R E S}{ }^{b}$ (Fig. 3(b)) given in terms of the $\Delta H_{1 / 2}$ used for both lines.

Notice that the increase on either $\Delta \phi$ (Fig. 3(a)-bottom) or $\Delta H_{R E S}$ (Fig. 3(b)-bottom) increases the signal amplitude in the $Y$ channel. However, increasing $\Delta \phi$ does not affect the lineshapes (Fig. 3(a)). On the other hand, both lineshapes in the $X$ and $Y$ channels are affected by increasing $\Delta H_{R E S}$ (Fig. 3(b)) especially when $\Delta H_{R E S}$ approaches $0.5 \Delta H_{1 / 2}$.

The model allows the analysis of other more complicated situations not shown here.

\section{B. High modulation frequency (HMF) and one resonant spin line}

In the special case of HMFs, where $B / G_{0}>0.01$, the current through the capacitor $C$ in Fig. 1(b) is responsible for two main features: (i) it attenuates the measured signal, $S$, acting as a low-pass filter, represented by $\beta$ in Eq. (9); and (ii) it promotes dynamic phase-shifts of the signal, see Eq. (8).

The most important feature for the quadrature detection is that $\theta$ represents a constant projection angle in Eqs. (11) and (12), while $\Phi\left(H_{L}\right)$ in Eq. (8) is a field dependent parameter, which varies from $\Phi_{0}$ to $\Phi_{P}$ in the same way that $G\left(H_{L}\right)$ varies from $G_{0}$ to $G_{0}+G_{H}$ in the resonance condition. In the case of only one RSL with constant phase $\phi$, it implies that

$$
\Phi_{0}=\phi+\tan ^{-1}\left(B / G_{0}\right),
$$

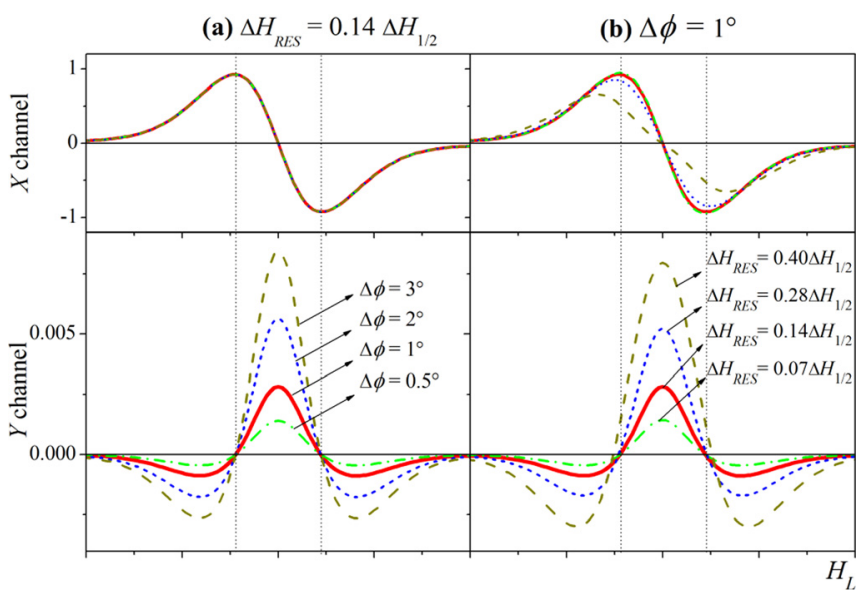

FIG. 3. Simulated EDMR signal in the $X$ and $Y$ channels of a dual phase lock-in amplifier for a similar condition as in Fig. 2(a). In (a), $\Delta \phi$ is varied from $0.5^{\circ}$ to $3^{\circ}$ and in (b) $\Delta H_{R E S}$ is varied from $0.07 \Delta H_{1 / 2}$ to $0.4 \Delta H_{1 / 2}$. 


$$
\Phi_{P}=\phi+\tan ^{-1}\left(B /\left(G_{0}+G_{H}\right)\right) .
$$

Thus, even if only a single RSL is present, there is no $\theta$ in which the EDMR signal has no projection at the $Y$ channel. Figure 4(a) shows an example of a quadrature EDMR signal for a single RSL $\left(G_{H}>0\right)$ where $B / G_{0}=0.01$ and $G_{H} / G_{0}=0.02$. The amplitudes are normalized by the maximum in the $X$ channel. The signal in the $Y$ channel is shown using different $\theta$ from $\Phi_{0}$ to $\Phi_{P}$. For this or any similar condition, variation in $\theta$ in this range does not noticeably affect the signal in the $X$ channel.

Notice that in Fig. 4(a), the minimum amplitude in $Y$ is reached when $\theta \approx\left(\Phi_{0}+\Phi_{P}\right) / 2=\Phi_{M}$ as expected. In this case a non-ordinary line-shape that resembles the third derivative of $G$ or the second derivative of the signal in the $X$ channel is observed.

Figure 4(b) shows the simulated EDMR signal amplitude at channels $X$ and $Y$ as a function of $B / G_{0}$, using $\theta=\Phi_{M}$ and $G_{H} / G_{0}=0.02$. The dependence of the signal in the $X$ channel is of a low pass filter as expected, Eq. (10). The behavior of the signal in the $Y$ channel is dominated by $\Phi_{0}-\Phi_{P}$ but $\beta$ plays a relevant role. The ratio of the amplitude of the signals in the $Y$ and $X$ channels reaches a maximum when $B \approx G_{0}$ (or equivalently $\Phi_{0} \approx \phi+45^{\circ}$ ), close to the attenuation threshold, i.e., when the signal in the $X$ channel starts to be attenuated. To give a numerical example, this situation is reached at $f=10 \mathrm{kHz}$ for $R=1 / G_{0}=100 \mathrm{k} \Omega$ and $C=1 \mathrm{nF}$.

One important feature of the HMF regime is that one can distinguish the sign of the signal, quenching $\left(G_{H}<0\right)$ or enhancing $\left(G_{H}>0\right)$, see Fig. 4(c). As expected, the signal in the $X$ channel changes sign when the EDMR signal changes from enhancing to quenching, due to Eq. (9) and the even nature of the cosine function in Eq. (11). However, the signal in the $Y$ channel in the same situation does not change sign due to the odd nature of the sine function in Eq. (12).

Considering the above delineated features, it is possible to tune the modulation frequency, or the total circuit capacitance in order to be in the HMF regime and thus determine the sign of the EDMR signal. However, this procedure/ interpretation must be carefully tested, since as discussed in Lee's work the phase response of the RSLs in EDMR depends on several parameters. ${ }^{26}$ In addition, it has also been observed by pulsed techniques that quenching and enhancing signals can be observed in the same sample on different time scales. $^{14}$

Note that measurements in the HMF regime, as described in Fig. 4(a), could mask the signal due two RSLs with different phases, undermining the discrimination of the individual RSLs. This will become clearer in Sec. IV.

\section{APPLICATION TO EXPERIMENTAL DATA}

In this section, we apply our model to representative results of EDMR found in the literature. For this purpose, the fitting program RESONA was used, see Appendix B.

\section{A. $\mathrm{Alq}_{3}$ based OLEDs}

Figures 5(a) and 5(b) show the EDMR spectra as observed in the $X$ and $Y$ channels of a PSD system for an undoped OLED at room temperature under the same experimental conditions but different modulation frequencies, in (a) $f=13 \mathrm{~Hz}$ and (b) $f=13 \mathrm{kHz}$. This device has the following structure $\mathrm{Al} / \mathrm{LiF} / \mathrm{Alq}_{3} / \alpha-\mathrm{NPD} / \mathrm{CuPc} / \mathrm{ITO}$, details can be found in Ref. 25. Figure 5(c) shows the quadrature EDMR spectrum from a DCM-TPA doped OLED at $\mathrm{T}=110 \mathrm{~K}$ with $f=133 \mathrm{~Hz}$. In this case, the structure is $\mathrm{Al} / \mathrm{LiF} / \mathrm{Alq}_{3} / \mathrm{DCM}-\mathrm{TPA} / \alpha-\mathrm{NPD} /$ $\mathrm{CuPc} / \mathrm{ITO}$, see Refs. 25 and 33. The red lines were obtained by fitting using our model. The blue dashed line in Fig. 5(b) was obtained using the fit parameters of Fig. 5(a). Table I shows the parameters obtained experimentally and the best fits obtained.

Notice that the amplitude of the signal in the $X$ channel decreases strongly from $f=13 \mathrm{~Hz}$ (Fig. 5 (a)) to $f=13 \mathrm{kHz}$ (Fig. 5(b)), but there is no observable change in the lineshape. The opposite occurs for the signal in the $Y$ channel: the maximum amplitude remains practically the same $(\sim 1 \mu \mathrm{V})$ but the lineshape changes significantly. Both features can be explained based on what was discussed in Sec. III B. For $f=13 \mathrm{~Hz}$ (Fig. 5(a)), $B / G_{0}<0.01$, thus the signal is in the LMF regime. From our model, the signal in the $Y$ channel can only be explained by the contribution of two RSLs with different phases with respect to the reference. On the other hand, for $f=13 \mathrm{kHz}$ (Fig. 5 (b)), $B / G_{0} \sim 0.05$, the measurements are in the HMF regime.
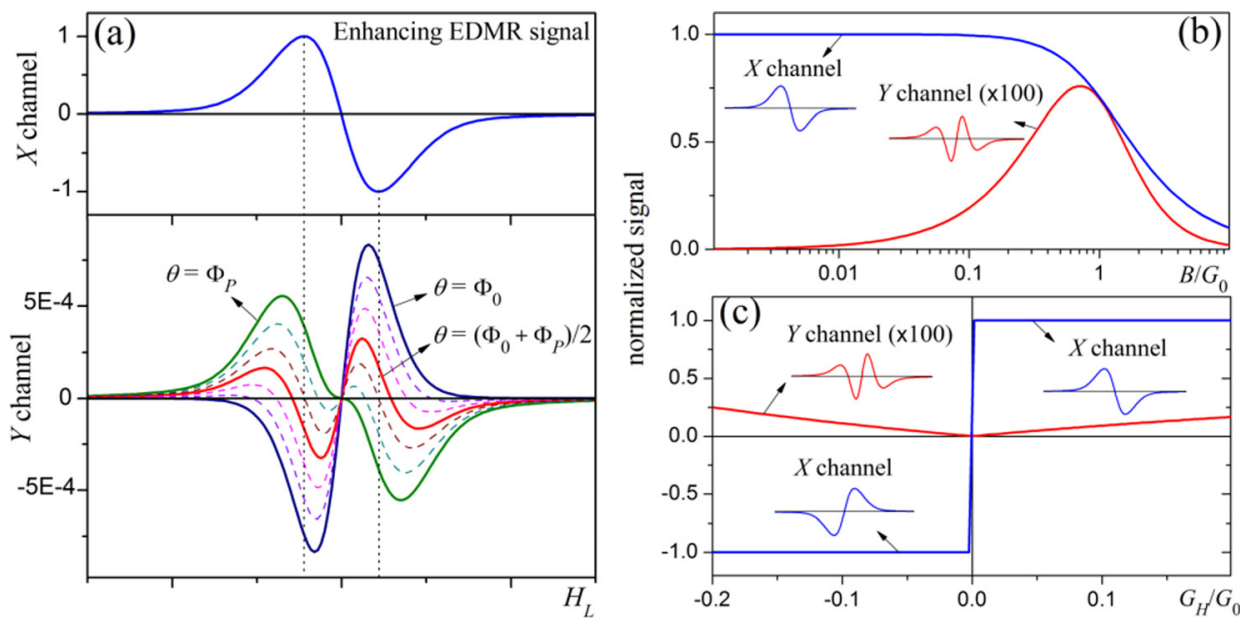

FIG. 4. (a) Simulated EDMR signal for $\quad G_{H}>0, \quad B / G_{0}=0.01, \quad$ and $G_{H} / G_{0}=0.02$. The amplitude is normalized by the maximum of the signal in the $X$ channel. The curves are obtained for different $\theta$ from $\Phi_{0}$ to $\Phi_{P}$; (b) Normalized signal amplitude in the $X$ and $Y$ channels as a function of $B / G_{0}$ for $G_{H} / G_{0}=0.02$ and $\theta=\Phi_{M}$; (c) Normalized signal amplitude in the $X$ and $Y$ channels as a function of $G_{H} / G_{0}$ for $B / G_{0}=0.01$, and $\theta=\Phi_{M}$. 
(a) Undoped OLED $\left(f_{m}=13 \mathrm{~Hz}\right)$

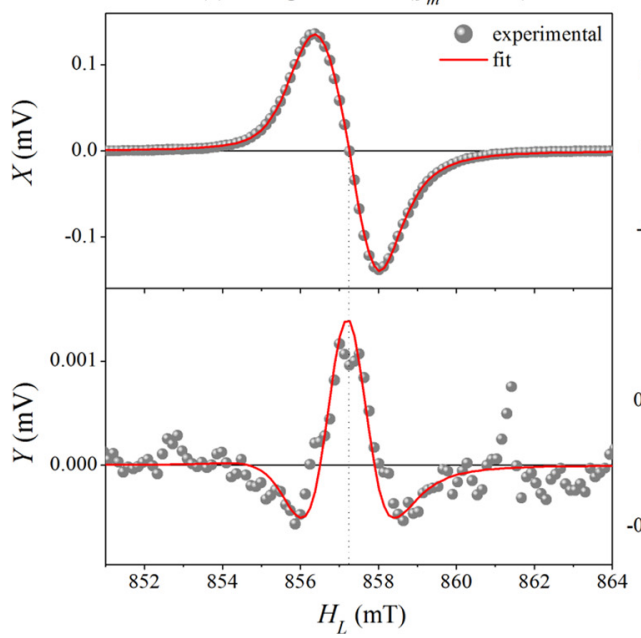

(b) Undoped OLED $\left(f_{m}=13 \mathrm{kHz}\right)$

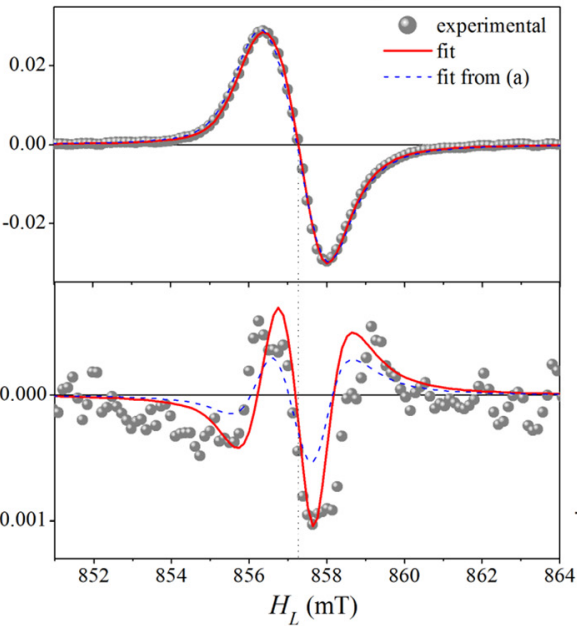

(c) Doped OLED $\left(f_{m}=133 \mathrm{~Hz}\right)$

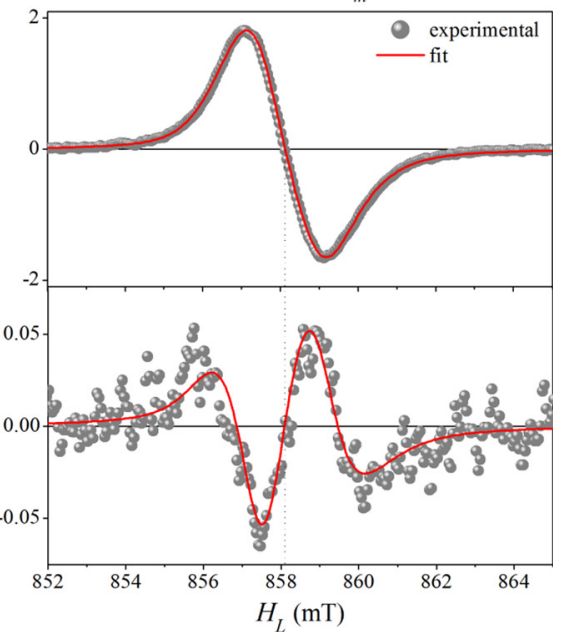

FIG. 5. Simulation (red lines) of experimental EDMR spectra (gray points) for an undoped Alq ${ }_{3}$ OLEDs using different magnetic field modulation frequency: (a) $f=13 \mathrm{~Hz}$ and (b) $f=13 \mathrm{kHz}$. Measurements were done at room temperature. In (c), the results using a doped OLED at $110 \mathrm{~K}$ with $f=133 \mathrm{~Hz}$ are presented. The blue dashed line in (b) uses the same fitting parameters of (a).

The strength of the proposed model can be better realized by its ability to describe EDMR spectra taken from the same device in two different modulation frequencies, $13 \mathrm{~Hz}$ in Fig. 5(a), and $13 \mathrm{kHz}$ in Fig. 5(b), using the same fitting parameters. In fact the blue dashed line in Fig. 5(b) is the EDMR signal predicted by the model from the fitted parameters on the experimental signal of Fig. 5(a). It was obtained by using the overall capacitance of $C=0.52 \mathrm{nF}$, which renders $\beta=1$ (no attenuation) for $f=13 \mathrm{~Hz}$ and $\beta=0.23$ for $f=13 \mathrm{kHz}$ in Eq. (10). To get the best fit on Fig. 5(b) (red curve), however, the overall capacitance was increased to $C=0.82 \mathrm{nF}$, rendering $\beta=0.11$ for $f=13 \mathrm{kHz}$, consequently the overall signal amplitude measured by the absolute value $G_{H}{ }^{a} / G_{0}$ and $G_{H}{ }^{b} / G_{0}$ has also to be increased for this case, see Table I. This is an indication that the absolute values of $G_{H} / G_{0}$ can differ from the real variation of the sample's conductivity $(\Delta \sigma / \sigma)$, as well as the capacitance value used to fit the data can differ from the real sample-plus-circuit capacitance, due to the simplicity of the equivalent circuit proposed. Moreover, it is well known that OLEDs cannot be completely described by simple RC circuits. In fact, impedance analyses show that $R$ and $C$ parameters present a significant dependence with frequency, including the existence of "negative capacitance" in low frequency regime, which is associated to charge carriers relaxation processes. ${ }^{35-37}$

As can be seen, the proposed circuit model is able to determine fundamental spectroscopic parameters of each RSL with relatively high precision and reliability. In Table I for the undoped OLED, the values obtained from the fitting, except for $G_{H} / G_{0}$, are very close for the two frequencies under analysis. The new set of $g$-values is close to the earlier determined values of Ref. 25 for $f=133 \mathrm{~Hz}$. On the other

TABLE I. Fitting parameters from the present model (red line of Fig. 5) compared to the values found in the literature. $\Delta H_{p p}$ were calculated from $\Delta H_{1 / 2}{ }^{*}$. $1 / G_{0}$ values are obtained by dividing the applied DC voltage $V_{0}$ by the measured DC current $I_{0}$ corrected by the measuring resistance $R_{M}$. The errors were evaluated by averaging the result of 3 or more independent fitting procedures. ( $\Delta H_{p p}=\Delta H_{1 / 2} / \delta, \delta$ varying linearly from $\sqrt{3}$ (Lorentzian) to $\sqrt{2 \ln 2}$ (Gaussian) considering $\alpha$ from Eq. (1).)

\begin{tabular}{|c|c|c|c|c|c|}
\hline \multirow[b]{2}{*}{ Parameter } & \multicolumn{3}{|c|}{ Undoped $\mathrm{Alq}_{3}$ OLED } & \multicolumn{2}{|c|}{ Doped $\mathrm{Alq}_{3}$ OLED } \\
\hline & Present work $(13 \mathrm{~Hz})$ & Present work $(13 \mathrm{kHz})$ & Ref. $25(133 \mathrm{~Hz})$ & Present work $(133 \mathrm{~Hz})$ & Ref. $33(133 \mathrm{~Hz})$ \\
\hline$g^{a}$ & $2.0028 \pm 0.0001$ & $2.0030 \pm 0.0002$ & $2.0028 \pm 0.0002$ & $2.0026 \pm 0.0003$ & 2.0039 \\
\hline$g^{b}$ & $2.0045 \pm 0.0001$ & $2.0044 \pm 0.0003$ & $2.0040 \pm 0.0001$ & $2.0049 \pm 0.0004$ & 2.0042 \\
\hline$\Delta g$ & $0.0017 \pm 0.0002$ & $0.0013 \pm 0.0004$ & $0.0012 \pm 0.0003$ & $0.0023 \pm 0.0005$ & 0.0003 \\
\hline$\Delta H_{p p}{ }^{a}(\mathrm{mT})$ & $1.03 \pm 0.05$ & $1.00 \pm 0.02$ & $2.0-3.4$ & $1.51 \pm 0.05$ & $1.82 \pm 0.01$ \\
\hline$\Delta H_{p p}^{b}(\mathrm{mT})$ & $1.10 \pm 0.03$ & $1.11 \pm 0.03$ & 1.5 & $1.46 \pm 0.05$ & $3.0-4.2$ \\
\hline$\alpha^{a}$ & $0.14 \pm 0.11$ & $0.20 \pm 0.06$ & $\ldots$ & $0.16 \pm 0.07$ & $\ldots$ \\
\hline$\alpha^{b}$ & $0.61 \pm 0.05$ & $0.48 \pm 0.10$ & $\ldots$ & $0.51 \pm 0.12$ & $\ldots$ \\
\hline$G_{H}{ }^{a} / G_{0}$ & $-0.079 \pm 0.006$ & $-0.18 \pm 0.01$ & $\ldots$ & $0.145 \pm 0.013$ & $\ldots$ \\
\hline$G_{H}^{b} / G_{0}$ & $-0.055 \pm 0.003$ & $-0.11 \pm 0.01$ & $\ldots$ & $0.154 \pm 0.010$ & $\ldots$ \\
\hline$\Delta \phi\left({ }^{\circ}\right)$ & $1.2 \pm 0.2$ & $1.3 \pm 0.5$ & $0.4-2.8$ (Ref. 34) & $0.4 \pm 0.3$ & $\ldots$ \\
\hline $1 / G_{0}(\mathrm{k} \Omega)$ & 45.7 & 42.6 & 45.7 & 790 & 790 \\
\hline$R_{M}(\mathrm{k} \Omega)$ & 0.1 & 0.1 & 0.1 & 10 & 10 \\
\hline$H_{m}(\mathrm{mT})$ & 0.5 & 0.5 & 0.5 & 0.5 & 0.5 \\
\hline$C(\mathrm{nF})$ & 0 & 0.82 & $\ldots$ & 1.8 & $\ldots$ \\
\hline
\end{tabular}


hand, the values for $\Delta H_{p p}$ obtained from the fits are systematically smaller than the experimental values in all cases. This could be related to the fact that $\Delta H_{p p}$ in the case of our model were calculated from the fitted $\Delta H_{1 / 2}$, while the data from the literature were determined directly from the experimental curves in the $Y$ channel using arbitrary phases. Moreover, the slight line broadening effect due the modulated field $H_{m}, H_{m} \sim \Delta H_{p p} / 2$, is considered by the model. In fact, by ignoring this correction, the model returns $\Delta H_{p p}$ values $10 \%$ to $20 \%$ higher than those shown in Table I. As discussed before, assuming that in Fig. 5(b), the signal is in the HMF regime, the EDMR signal represents a decrease in conductivity during resonance (quenching), so the values for $G_{H}{ }^{a}$ and $G_{H}{ }^{b}$ are negative in Table I. Regarding the signal linewidth, it is important that similar $\Delta H_{p p}$ values are obtained for undoped OLEDs at different modulation frequencies (see values in Table I). In fact, since EDMR linewidths for organic semiconductors are mainly associated to unresolved hyperfine splittings, they are not supposed to be sensitive to the modulation frequency.

For the doped OLED (Fig. 5(c)), the $g$-values obtained from the fit are quite different from the previous reported values. ${ }^{33}$ The $g$-factor difference $(\Delta g)$ between the two RSLs is larger for the doped OLED $(\Delta g=0.0023)$ than for the undoped OLED $(\Delta g \sim 0.0015)$. On the other hand, the phase difference $(\Delta \phi)$ is higher for the undoped OLED $\Delta \phi \sim 1^{\circ}$, against $\Delta \phi \sim 0.2^{\circ}$. However, it is worth to emphasize that, differently from $g$ and $\Delta H_{p p}, \Delta \phi$ is a dynamic parameter ${ }^{26}$ and is probably strongly dependent on the experimental conditions. Thus, for a better discussion regarding $\Delta \phi$, we would need a systematic study varying experimental parameters, such as temperature or microwave power. However, the relatively low values of $\Delta \phi$ reinforce the interpretation presented in the Refs. 22, 25, and 33 where EDMR signal of $\mathrm{Alq}_{3}$ based OLEDs are due to one SDP with two spin pairs (electron and holes) experiencing slight different chemical environments.

As mentioned before, the transition from LMF regime to HMF is strongly dependent on the experimental conditions. This feature is indeed observed in the case of the doped OLED, due to the low temperature used, a relative high resistance $\left(1 / G_{0}=790 \mathrm{k} \Omega\right)$ combined with a higher capacitance $(1.8 \mathrm{nF})$ results in a HMF regime even at $f=133 \mathrm{~Hz}$. In this case, the comparison between the lineshapes of the signal at $X$ and $Y$ channels suggest an enhancing EDMR response $\left(G_{H}>0\right)$ as revealed in Fig. 4(c). As already pointed out in Sec. III B, in HMF, the discrimination of the individual RSLs is undermined. This leads to imprecise determinations of $g$-factors and $\Delta H_{p p}$ values.

It is important to emphasize the relevance of the analysis considering both regimes, LMF and HMF, which respectively allows the determination of spectroscopic parameters of distinct RSLs in the system and the nature of the dominating signal (quenching or enhancing). For example, the signals obtained in the HMF regime for undoped and doped OLEDS suggest the existence of distinct mechanisms in these devices responsible, respectively, for a quenching and enhancing EDMR signals. As shown by Lee et al., ${ }^{26}$ it is very difficult to access the exact nature of the SDP

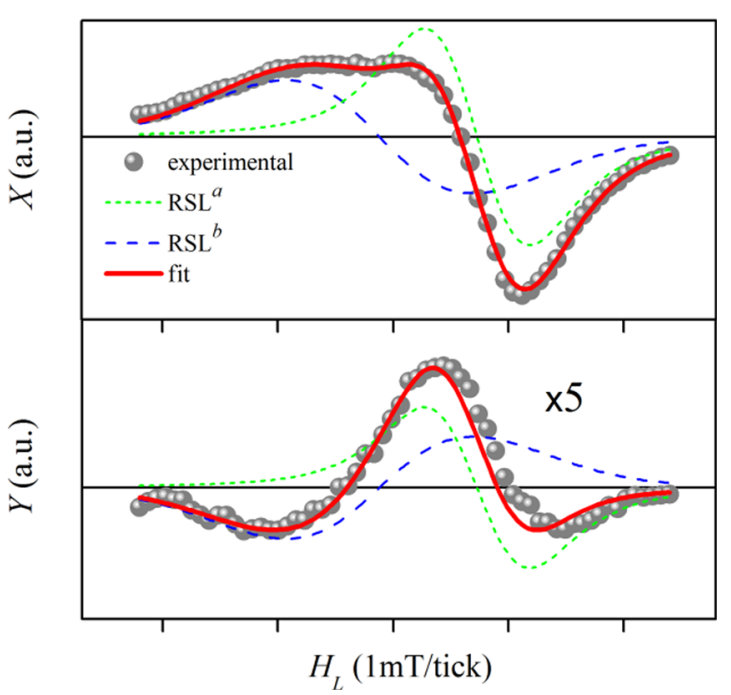

FIG. 6. EDMR spectra of a-Si:H adapted from Ref. 21 shown in gray. The solid red line is the best fit using our model, together with its components, $\mathrm{RSL}^{a}$ in dotted green and $\mathrm{RSL}^{b}$ in dashed blue.

responsible for each signal, however, based on a classical interpretation the quenching signal of undoped OLED is compatible to an increase on the singlet exciton population induced by ESR condition, while the enhancing signal of doped OLED could be associated to a detrapping processes. ${ }^{15}$

\section{B. a-Si:H}

In order to further explore the applicability of the circuit model, EDMR results from a-Si:H obtained by Dersch et $a l .{ }^{20}$ were analyzed. As already discussed, two independent SDPs were identified in a-Si:H. ${ }^{20}$ The experimental quadrature EDMR spectra used in this section was built from published data, and thus the accuracy is limited compared to Sec. IV A.

Figure 6 shows the reconstructed EDMR spectra in gray, taken from Figure 6 of Ref. 20. The solid red line shows the resulting fitting curves composed by the sum of the individual RSL shown as dashed blue and dotted green lines. Table II shows the parameters obtained from the fitting process.

In this example, the fitting process was done with the limited experimental details available. For a more precise analysis, the complete set of experimental parameters is

TABLE II. Parameters used to fit the EDMR spectra from a-Si: $\mathrm{H}^{20}$ using the proposed model as well as the corresponding published data.

\begin{tabular}{lcc}
\hline \hline \multirow{2}{*}{ Parameter } & \multicolumn{2}{c}{$\mathrm{a}-\mathrm{Si}: \mathrm{H}$} \\
\cline { 2 - 3 } & Present work & Ref. 20 \\
\hline$g^{a}$ & $2.0051 \pm 0.0003$ & 2.005 \\
$g^{b}$ & $2.0100 \pm 0.0005$ & 2.01 \\
$\Delta H_{p p}{ }^{a}(\mathrm{mT})$ & $0.69 \pm 0.05$ & 0.6 \\
$\Delta H_{p p}{ }^{b}(\mathrm{mT})$ & $1.51 \pm 0.09$ & 1.2 \\
$\alpha^{a}$ & $0.15 \pm 0.05$ & $\ldots$ \\
$\alpha^{b}$ & $0.75 \pm 0.11$ & $\ldots$ \\
$\Delta \phi\left({ }^{\circ}\right)$ & $16.6 \pm 0.9$ & $\sim 15$ \\
\hline \hline
\end{tabular}


needed. Notice also that the model uses the lineshape of a mixture of symmetric Lorentzian and Gaussian functions, see Eq. (1), which do not correspond always to real situations, especially in inorganic materials.

Despite the limitations above described, Fig. 6 clearly shows that the proposed circuit model was able to fit the aSi:H EDMR signal with a high degree of accuracy, and the fitting parameters are quite close to those previous published, see Table II.

The discussion of the results presented in Table II is not in the scope of this work, but it is interesting to notice the existence of a higher value of $\Delta \phi$, in comparison to those determined for $\mathrm{Alq}_{3}$ based OLEDs. This discrepancy is compatible with the interpretation that a-Si:H signals are related to two SDPs, while $\mathrm{Alq}_{3}$ OLEDs signals are associated just to one SDP with distinguishable precursor pair partners. Indeed, weaker phase correlation could be expected in the response of RSLs coming from independent processes, allowing the observation of significant phases difference in the case of a-Si:H. On the other hand, given the stronger exchange interaction between electron-hole pairs, smaller values of $\Delta \phi$ are expected for $\mathrm{Alq}_{3}$ based OLEDs.

The origin of different phases between the SDPs or spin pairs is still not well understood. In our model, it is a free parameter essential to describe the EDMR out of phase signal; however at this moment, we are not able to describe its nature. In principle, it could be associated to distinct relaxation times or any other dynamic parameter related to transport/recombination processes.

In summary, in this work, we provide a circuit model that allows the extraction and evaluation of EDMR parameters with high accuracy and reproducibility. Two distinct regimes were identified depending on the magnetic field modulation frequency. These regimes define two distinct response domains, LMF and HMF, which carry complementary information about the system under study. In the LMF regime, the non-vanishing signal in the $Y$ channel is dominated by the dynamics of RSLs, allowing the isolation of relevant spectroscopic parameters, associated to distinct SDPs and/or spin pairs. In the HMF regime, the signal in the $Y$ channel can be dominated by the circuit capacitance. In this regime, it is shown that it is possible to discriminate whether the EDMR signal is due to an increase or a decrease in the sample conductivity.

Moreover, the proposed circuit model is constituted by simple mathematical equations, which allows its numerical implementation. In fact, a computer program called RESONA was developed in order to analyze and fit experimental quadrature EDMR spectra. The RESONA program, see Appendix $\mathrm{B}$, includes unique features as line broadening correction due to over modulation, see Appendix A, and phase analysis by evaluating the entire quadrature EDMR signal in individual $X$ and $Y$ channels from PSD measurements.

\section{CONCLUSION}

A model for EDMR based on an equivalent electrical circuit is presented. The proposed model is able to describe and predict a variety of complex quadrature EDMR signals with non-vanishing components in the $Y$ channel. Two different regimes have been described depending on the magnetic field modulation frequency. In the low modulation frequency regime, the model is able to isolate, discriminate, and characterize individual contributions of distinct resonant spin lines. In the high modulation frequency regime, it is possible to determine whether the EDMR signal is coming from an increase or decrease in the sample conductivity under resonance.

The model was implemented as a software and successfully applied to experimental data taken from the literature. The fitting process allows not only the determination of accurate spectroscopic values, as $g$-factor and $\Delta H_{p p}$ of the individual resonant lines, but also resolves the phase difference between them. The fitting program implemented called RESONA is computationally fast, typically it takes a few minutes to fit the data in a standard PC.

\section{ACKNOWLEDGMENTS}

This work was financially supported by the Brazilian agencies CAPES (Proc. 23038.008351/2010-17), FAPESP (Proc. 2012/03116-7, 2011/21830-6, and 2008/57872-1 INCTMN) and CNPq. We are also thankful to Professor F. Nüesch and Professor L. Zuppiroli for providing the $\mathrm{Alq}_{3}$ based OLEDs.

\section{APPENDIX A: LINE BROADENING DUE TO MAGNETIC FIELD MODULATION}

The aim of this appendix is to provide a mathematical formula to simulate the line broadening effects due to magnetic field modulation on EDMR spectra. The approximations used are made in order to generate formulas that are easily implemented computationally.

From a mathematical point of view, in PSD, the output signal is obtained by multiplying the input signal $V_{M}$ by the reference signal $(\sin \omega t)$ and then integrating over the time constant $T C$. Considering this, $c_{1}$ in Eq. (9) can be written as

$$
c_{1}\left(H_{L}\right)=\frac{1}{T C} \int_{t_{i}}^{t_{i}+T C} G(t) \sin \omega t \mathrm{~d} t, \quad \mathrm{i}=0,1,2, \ldots, \Delta t / T C,
$$

where the measuring time $t_{i}$ is related to $H_{L}$ by the following relation derived from Eq. (2):

$$
H_{L}=H_{0}+\Delta H\left(\frac{t_{i}+T C / 2}{\Delta t}-\frac{1}{2}\right) .
$$

For low speed field sweeps, $\Delta H / \Delta t \ll 2 H_{m} f$, the variation of $H_{L}$ within $T C$ can be disregarded, i.e., $H_{L}$ can be considered constant from $t_{i}$ to $t_{i}+T C$. Thus, $c_{1}$ can be approximated to 


$$
c_{1}\left(H_{L}\right)=\frac{1}{T C} \int_{t_{i}}^{t_{i}+T C} G\left(H_{L}+H_{m} \sin \omega t\right) \sin \omega t \mathrm{~d} t,
$$

where the phase difference between $G$ and the field modulation was set to zero, since we are only interested in the modulus of the signal.
Equation (A3) thus returns the effective or average slope of $G\left(H_{L}\right)$ within $H_{L}-H_{m}$ and $H_{L}+H_{m}$, which is supposed to be independent of $T C$ when $T C \ll \Delta t$ and $T C=2 \pi k / \omega$ with $k=1,2,3, \ldots$ Therefore, choosing $k=1, T C=2 \pi / \omega$, i.e., $T C$ covers one single modulation cycle. Using $\xi=\omega t$, one can write

$$
\begin{aligned}
c_{1}\left(H_{L}\right) & =\frac{1}{2 \pi} \int_{0}^{2 \pi} G\left(H_{L}+H_{m} \sin \xi\right) \sin \xi \mathrm{d} \xi \\
& =\frac{1}{2 \pi}\left(\int_{0}^{\pi} G\left(H_{L}+H_{m} \sin \xi\right) \sin \xi \mathrm{d} \xi+\int_{\pi}^{2 \pi} G\left(H_{L}+H_{m} \sin \xi\right) \sin \xi \mathrm{d} \xi\right) \\
& =\frac{1}{2 \pi}\left(\int_{0}^{\pi} G\left(H_{L}+H_{m} \sin \xi\right) \sin \xi \mathrm{d} \xi-\int_{0}^{\pi} G\left(H_{L}-H_{m} \sin \xi\right) \sin \xi \mathrm{d} \xi\right) \\
& =\frac{1}{\pi} \int_{0}^{\pi / 2}\left[G\left(H_{L}+H_{m} \sin \xi\right)-G\left(H_{L}-H_{m} \sin \xi\right)\right] \sin \xi \mathrm{d} \xi .
\end{aligned}
$$

In Eq. (A4), $c_{1}$ is a continuous function of $H_{L}$ that can be numerically calculated by different commercial softwares. Another possibility is to use the following:

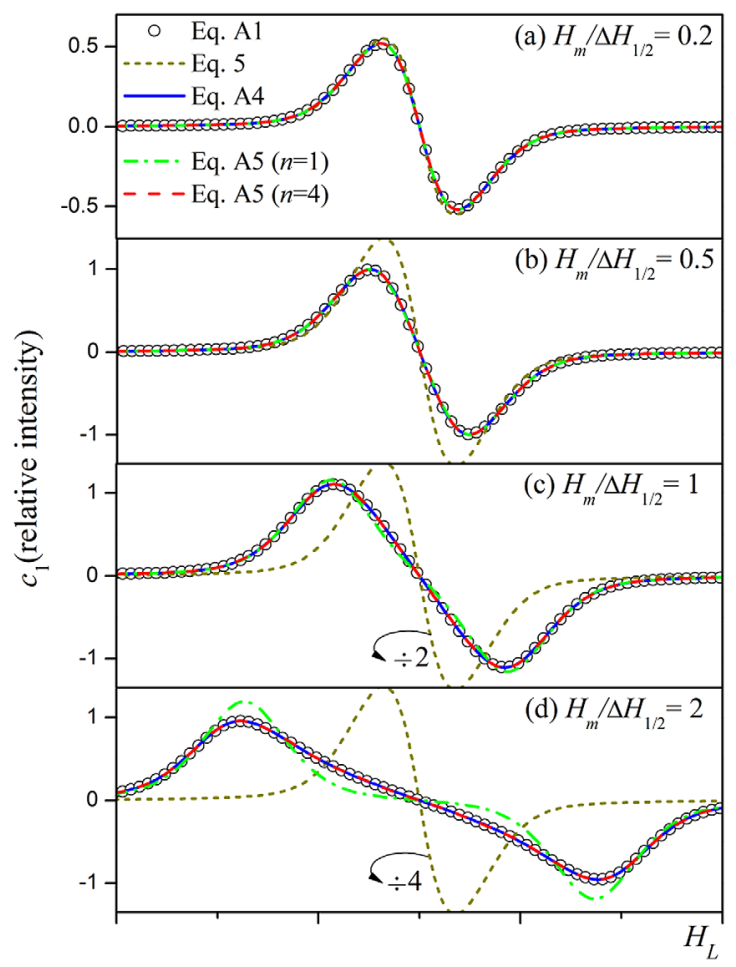

FIG. 7. Simulated EDMR signal obtained from different equations of $c_{1}$ under different magnetic field modulation conditions: (a) $H_{m} / \Delta H_{1 / 2}=0.2$; (b) $H_{m} / \Delta H_{1 / 2}=0.5$; (c) $H_{m} / \Delta H_{1 / 2}=1$; and (d) $H_{m} / \Delta H_{1 / 2}=2$. The open circles represent the signal using Eq. (A1). The blue dashed line was obtained using Eq. (5), while the blue solid line Eq. (A4). The green dashed-dotted line used Eq. (A5) with $n=1$, while the red dashed line $n=4$.

$$
\begin{aligned}
c_{1}\left(H_{L}\right)= & \frac{1}{2 n+1} \sum_{i=1}^{n}\left[G\left(H_{L}+H_{m} \sin \frac{i \pi}{2 n+1}\right)\right. \\
& \left.-G\left(H_{L}-H_{m} \sin \frac{i \pi}{2 n+1}\right)\right] \sin \frac{i \pi}{2 n+1},
\end{aligned}
$$

where $n$ is chosen as the next integer of $2 H_{m} / \Delta H_{1 / 2}$, i.e.: if $H_{m} / \Delta H_{1 / 2} \sim 1$, one uses $n=2$; if $H_{m} / \Delta H_{1 / 2} \sim 2$, uses $n=4$; and so forth.

In Fig. 7, a comparison between various simulations are presented for different magnetic field modulation conditions. For $H_{m} / \Delta H_{1 / 2} \leq 0.2$, the EDMR signal amplitude is directly proportional to $H_{m}$, and there is no significant line broadening due to $H_{m}$. Thus for $H_{m} / \Delta H_{1 / 2} \leq 0.2$, Eq. (5) is a good approximation. However, for $H_{m} / \Delta H_{1 / 2} \geq 0.5$ shown in Figures 7(b)-7(d), the signal amplitude is independent of $H_{m}$ and there is significant line-shape broadening. Thus, signal derived from Eq. (5) is no longer valid and the use of Eq. (A4) or Eq. (A5) is needed.

It is clear from Fig. 7 that the use of Eq. (A5) with $n=4$ is a good approximation for $H_{m} \leq 2 \Delta H_{1 / 2}$, and $n=1$ for $H_{m}<\Delta H_{1 / 2}$. The later has the following expression:

$$
c_{1}\left(H_{L}\right) \approx \frac{\sqrt{3}}{6}\left[G\left(H_{L}+\frac{\sqrt{3}}{2} H_{m}\right)-G\left(H_{L}-\frac{\sqrt{3}}{2} H_{m}\right)\right] .
$$

\section{APPENDIX B: THE FITTING PROGRAM-RESONA}

The proposed circuit model described above is mathematically simple and allowed its implementation in a software named RESONA. The computational routine has a 
user-friendly interface allowing the operator to continuously modify each parameter and visualize the simulated and experimental data in two screens that represent the quadrature signal, $X$ and $Y$ channels, in real time, with the respective sum of squared residuals.

RESONA is developed in Agilent VEE and is available for free at https://sites.google.com/site/resonaproject. To run the program, it is only necessary to download and install the Agilent VEE Runtime program available for free at http://www.agilent.com.

The program has a single interface panel that allows the input of the experimental parameters. Since commonly the magnetic field must be corrected, the program has this feature. All the simulation parameters are available using slide bars, and the main results are shown both graphically and in tables. A quick users guide is available by pressing the button "Info."

The program has also an automatic fitting option that minimizes the sum of squared residuals of one or both graphs to get a fine adjustment of the model parameters. It takes 1 to $5 \mathrm{~min}$ to reach a good fit reproducibly. The values of the parameters employed and the resulting fitting curves can be saved. This program can also be used for ESR spectra simulation.

${ }^{1}$ C. J. Cochrane, P. M. Lenahan, and A. J. Lelis, J. Appl. Phys. 109, 014506 (2011).

${ }^{2}$ M. S. Dautrich, P. M. Lenahan, and A. J. Lelis, Appl. Phys. Lett. 89, 223502 (2006).

${ }^{3}$ D. R. McCamey, G. W. Morley, H. A. Seipel, L. C. Brunel, J. van Tol, and C. Boehme, Phys. Rev. B 78, 045303 (2008).

${ }^{4}$ D. J. Meyer, P. M. Lenahan, and A. J. Lelis, Appl. Phys. Lett. 86, 023503 (2005).

${ }^{5}$ G. Li, C. H. Kim, P. A. Lane, and J. Shinar, Phys. Rev. B 69, 165311 (2004).

${ }^{6}$ V. Dyakonov, N. Gauss, G. Rösler, S. Karg, W. Rieß, and M. Schwoerer, Chem. Phys. 189, 687 (1994).

${ }^{7}$ P. A. Lane, L. S. Swanson, Q. X. Ni, J. Shinar, J. P. Engel, T. J. Barton, and L. Jones, Phys. Rev. Lett. 68, 887 (1992).

${ }^{8}$ D. R. McCamey, H. Huebl, M. S. Brandt, W. D. Hutchison, J. C. McCallum, R. G. Clark, and A. R. Hamilton, Appl. Phys. Lett. 89, 182115 (2006).

${ }^{9}$ C. F. O. Graeff, M. S. Brandt, M. Stutzmann, M. Holzmann, G. Abstreiter, and F. Schäffler, Phys. Rev. B 59, 13242 (1999).

${ }^{10}$ C. G. Yang, E. Ehrenfreund, and Z. V. Vardeny, Phys. Rev. Lett. 99, 157401 (2007).
${ }^{11}$ D. M. Hofmann, D. Kovalev, G. Steude, B. K. Meyer, A. Hoffmann, L. Eckey, R. Heitz, T. Detchprom, H. Amano, and I. Akasaki, Phys. Rev. B 52, 16702 (1995).

${ }^{12}$ G. Kawachi, C. F. O. Graeff, M. S. Brandt, and M. Stutzmann, Phys. Rev. B 54, 7957 (1996).

${ }^{13}$ G. B. Silva, L. F. Santos, R. M. Faria, and C. F. O. Graeff, Phys. B: Condens. Matter 308-310, 1078 (2001).

${ }^{14}$ A. Schnegg, J. Behrends, M. Fehr, and K. Lips, Phys. Chem. Chem. Phys. 14, 14418 (2012).

${ }^{15}$ M. Stutzmann, M. S. Brandt, and M. W. Bayerl, J. Non-Crystall. Solids 266-269, 1 (2000).

${ }^{16}$ J. Behrends, A. Schnegg, K. Lips, E. A. Thomsen, A. K. Pandey, I. D. W. Samuel, and D. J. Keeble, Phys. Rev. Lett. 105, 176601 (2010).

${ }^{17}$ L. S. Swanson, J. Shinar, A. R. Brown, D. D. C. Bradley, R. H. Friend, P. L. Burn, A. Kraft, and A. B. Holmes, Phys. Rev. B 46, 15072 (1992).

${ }^{18}$ C. G. Yang, E. Ehrenfreund, F. Wang, T. Drori, and Z. V. Vardeny, Phys. Rev. B 78, 205312 (2008)

${ }^{19}$ V. Dyakonov, G. Rösler, M. Schwoerer, and E. L. Frankevich, Phys. Rev. B 56, 3852 (1997).

${ }^{20}$ H. Dersch, L. Schweitzer, and J. Stuke, Phys. Rev. B 28, 4678 (1983).

${ }^{21}$ R. Müller, P. Kanschat, S. von Aichberger, K. Lips, and W. Fuhs, J. NonCrystall. Solids 266-269, 1124 (2000).

${ }^{22}$ C. F. O. Graeff, G. B. Silva, F. Nüesch, and L. Zuppiroli, Eur. Phys. J. E 18, 21 (2005).

${ }^{23}$ T. Sato, H. Yokoyama, and H. Ohya, Chem. Lett. 35, 1428 (2006).

${ }^{24}$ S. Schaefer, S. Saremi, J. Behrends, K. Fostiropoulos, K. Lips, and W. Harneit, Phys. Status Solidi B 246, 2844 (2009).

${ }^{25}$ J. Gómez, F. Castro, F. Nüesch, L. Zuppiroli, and C. Graeff, Appl. Phys. A 108, 727 (2012)

${ }^{26}$ S.-Y. Lee, S. Paik, D. R. McCamey, and C. Boehme, Phys. Rev. B 86, 115204 (2012).

${ }^{27}$ T. Wimbauer, M. S. Brandt, M. W. Bayerl, N. M. Reinacher, M. Stutzmann, D. M. Hofmann, Y. Mochizuki, and M. Mizuta, Phys. Rev. B 58, 4892 (1998).

${ }^{28}$ W. Brütting, S. Berleb, and A. G. Mückl, Org. Electron. 2, 1 (2001).

${ }^{29}$ J. Bisquert, Phys. Chem. Chem. Phys. 13, 4679 (2011).

${ }^{30}$ F. Fabregat-Santiago, G. Garcia-Belmonte, I. Mora-Sero, and J. Bisquert, Phys. Chem. Chem. Phys. 13, 9083 (2011).

${ }^{31}$ L. Burtone, J. Fischer, K. Leo, and M. Riede, Phys. Rev. B 87, 045432 (2013).

${ }^{32}$ O. E. Myers and E. J. Putzer, J. Appl. Phys. 30, 1987 (1959).

${ }^{33}$ F. A. Castro, G. B. Silva, F. Nuesch, L. Zuppiroli, and C. F. O. Graeff, Org. Electron. 8, 249 (2007).

${ }^{34}$ J. Gómez, "Electrically detected magnetic resonance study of organics electronics and optoelectronics devices," Ph.D. thesis (Department of Physics, FFCLRP, Universidade de São Paulo, 2009).

${ }^{35}$ G. Garcia-Belmonte, J. Bisquert, P. R. Bueno, and C. F. O. Graeff, Chem. Phys. Lett. 455, 242 (2008).

${ }^{36}$ H. H. P. Gommans, M. Kemerink, and R. A. J. Janssen, Phys. Rev. B 72, 235204 (2005).

${ }^{37}$ E. Ehrenfreund, C. Lungenschmied, G. Dennler, H. Neugebauer, and N. S. Sariciftci, Appl. Phys. Lett. 91, 012112 (2007). 